\title{
Proposta de Arquitetura para Distribuição de Conteúdos Nomeados em NovaGenesis com P4
}

\author{
José Rodrigo dos Santos ${ }^{1}$, Tibério Tavares Rezende ${ }^{1}$, \\ Thiago Bueno da Silva ${ }^{1}$, Elcio C. do Rosário ${ }^{1}$ e Antônio M. Alberti ${ }^{1}$ \\ ${ }^{1}$ ICT Lab - Instituto Nacional de Telecomunicações (INATEL) \\ Santa Rita do Sapucaí - MG - Brasil \\ joserodrigo@gec.inatel.br, \\ (tiberio; elcio.carlos) emtel.inatel.br, \\ thiagobuenodgea.inatel.br, alberti@inatel.br
}

\begin{abstract}
This paper presents a study on the packet routing methods and techniques employed in Information-Centric Networks (ICN). Some important points are raised from existing ICN projects and their implementations aiming to inspire the evolution of the NovaGenesis (NG) architecture, a Future Internet proposal, towards the ICN and P4.
\end{abstract}

Resumo. Este artigo apresenta um estudo dos métodos e técnicas de roteamento de pacotes que são utilizadas nas Information-Centric Networks (ICN). São levantados alguns pontos importantes de implementações de ICNs existentes que podem inspirar uma evolução da arquitetura NovaGenesis (NG), uma proposta de Internet do Futuro, na direção de integrar ICN e P4.

\section{Introdução}

O desenvolvimento do conceito de Information-Centric Networks (ICN) é um dos resultados significativos de diferentes atividades internacionais de pesquisa usando arquiteturas de Internet do Futuro [ICN 2017]. ICN introduz o uso de dados nomeados como o princípio básico da troca de informações. Neste contexto, os dados se tornam independentes da sua localização, do aplicativo utilizado, do armazenamento e/ou do meio de transporte empregado, permitindo o armazenamento em cache e a sua replicação na rede. Tais conceitos são empregados em diversos tipos de arquiteturas e paradigmas de comunicação, os quais são conhecidos sob diferentes termos e incluem, por exemplo, a proposta Network of Information (NetInf) [Potys 2016], Named Data Networking (NDN) [Luo 2015] e Publish/Subscribe Networking [Mays 2016].

Este trabalho apresenta uma análise crítica de quais paradigmas podem ser incorporados na arquitetura de Internet do Futuro NovaGenesis, e integrados ao conceito de Software Defined Networks (SDN) [Li and Chen 2015]. Para tanto, a Seção 2 apresenta alguns conceitos da arquitetura NovaGenesis (NG). Na Seção 3 são levantados alguns pontos importantes de implementações existentes em redes NDN. Já a Seção 4 se concentra em abordar a linguagem Programming Protocol-Independent Packet Processors (P4), enquanto a Seção 5 discute a sinergia entre as propostas da NG, ICN e P4 e, por fim, a Seção 6 aponta as principais conclusões do artigo. 


\section{NovaGenesis}

Em 2011, uma proposta emergente de arquitetura alternativa à Internet atual foi idealizada para satisfazer requisitos exponenciais de tráfego, conteúdo, dispositivos e mobilidade, considerando o estado da arte das tecnologias de computação e comunicação. Por meio deste estudo, iniciou-se o projeto NG [A. Alberti 2017], o qual pode ser descrito de uma maneira concisa como uma arquitetura convergente para troca, processamento e armazenamento de informações, composta de um sistema distribuído orientado a serviços que utiliza nomeação; ligações entre nomes, ou Naming Binding (NB); resolução de nomes e contratos para satisfazer necessidades e requisitos emergentes de serviços, incluindo a implementação em software de protocolos de modo a prover escalabilidade e segurança.

No que condiz à comunicação, ao encaminhamento e ao roteamento de pacotes são realizados com base em nomes naturais ou auto-verificáveis pelo Proxy/Gateway/Controller Service (PGCS), o qual se vale do uso de memória compartilhada para comunicação intra sistema operacional (SO) ou raw socket para comunicação inter SO. Além disso, emprega-se o Publish/Subscribe Service (PSS) para assinar NBs disponíveis em um domínio. Desta maneira, os NBs são recebidos no Hash Table Service (HTS) e armazenados no sistema de arquivos Linux e são acessados pelo Proxy/Gateway via comunicação inter-processos para especificar informações necessárias sobre o próximo salto ou o caminho completo. O serviço responsável por localizar o HTS mais próximo e resolver as indireções é conhecido com Generic Indirection Resolution Service (GIRS). Assim o core NG é composto por PGCS, PSS, GIRS e HTS.

\section{Named Data Networking}

Em Named Data Networking (NDN) o foco é o conteúdo e não os nós finais, origem ou destino, ou seja, o foco não está na localização [K. Lei 2018]. O objetivo é entregar os dados de um produtor a seu consumidor usando o próprio nome do conteúdo, para o encaminhamento de pacotes na rede.

Considerando a estrutura NDN, cada nó da rede mantém uma Pending Interest Table (PIT) com base nas Forwarding Information Base (FIB) e no Content Store (CS). Nesta comunicação, a função da tabela PIT é manter uma lista atualizada dos pedidos recebidos e uma lista de nonces, que são números gerados aleatoriamente pelo consumidor para cada pacote de interesse. Já a FIB mantém as associações entre nome e interface, semelhante ao FIB IP, porém contém prefixos de nomes e pode associar diversas interfaces ao mesmo prefixo. Assim, registra informações do plano de dados e de roteamento, possibilitando um encaminhamento adaptável, encaminhando um pacote a uma determinada interface ao encontrar algum nome inscrito no interesse. Por sua vez, o CS é um cache de conteúdo feito a nível de rede, no qual o roteador pode guardar dados que serão utilizados por outras solicitações.

NDN geralmente emprega dois tipos de pacotes nomeados de Interest e Data. O conceito de Interest condiz com o pedido de dados associado a apenas um tipo de conteúdo desejado e possuem um "nonce". O dispositivo que deseja alguma informação gera e emite os pacotes de interesse, os quais estão ligados a pedaços de informação. Quando um nó recebe um pacote de interesse, ele verifica o CS do conteúdo nomeado e este dado é encaminhado ao solicitante quando estiver disponível no cache local. Caso contrário, o dado não está disponível e a PIT é consultada para ver se algum conteúdo 
semelhante já foi solicitado. Caso não haja uma entrada na PIT, esta será criada e a FIB será atualizada com o caminho ou salto do interesse. Os pacotes do tipo Data transportam os dados nas redes NDN e os pacotes circulam assinados. Com estas informações, o nó é capaz de identificar se o dado é o correto.

\subsection{Open Shortest Path First for Named-data}

O protocolo de roteamento que encontra o Open Shortest Path First (OSPF) possui algumas características que o tornam um forte candidato para implementações de roteamento em NDN. O OSPF é um protocolo amplamente utilizado devido a facilidade de acesso ao seu código fonte e por possuir algumas formas de divulgação do estado do enlace que possibilitam a divulgação de conteúdo ou informação desejada. O OSPF usa o Link State Advertising (LSA) e o Opaque Link State Advertising (OLSA). Os LSAs são avisos que o nó OSPF envia a seus vizinhos comunicando, informando e formando a topologia da rede. Assim, os LSAs contém informações dos estados das conexões, como, por exemplo, as métricas utilizadas e quais interfaces de um nó estão ligadas ou desligadas. Apesar de existirem onze tipos de LSAs, o OSPF for Named-data (OSPFN) [Wang 2012] faz uso de apenas três tipos que são usados para estender os recursos do OSPF, permitindo a transmissão de quaisquer dados. Ademais, o OSPFN consegue realizar o roteamento baseado em nomes e fornecer inúmeros caminhos para todos os prefixos, tornando o roteamento NDN possível através das OLSAs. Um problema em relação ao uso de LSAs ou OLSAs é que quanto mais dados são trafegados, maior será a carga de processamento, resultando em maior consumo de CPU.

\subsection{Coordinated Routing and Caching}

Nas redes IPs, a possibilidade de realizar sumarização de rotas facilita as tomadas de decisões em todos os tipos de roteamento existentes e utilizados na atualidade. Entretanto, a implementação de sumarização de rotas através de nomes de conteúdos, referentes aos mesmos fornecedores ainda não é possível em NDN. Para replicar isso nessas redes, os nós NDN precisam ter uma capacidade superior a $10^{9}$ entradas em suas FIBs, mesmo que o hardware dos roteadores e comutadores atuais ainda não atendam esta demanda. Conclui-se que o cache em todos os nós NDN não é eficiente [Choi 2014]. Outro ponto negativo está presente nas alterações de cache quando estas informações são anunciadas aos outros nós NDN mediante a um novo conteúdo armazenado e/ou substituído. Baseado nas premissas acima, o Coordinated Routing and Caching (CoRC) [Choi 2014] enfatiza as relações dos Autonomous Systems (AS) dos roteamentos Inter-AS, Intra-AS e custo do volume de tráfego entre os ASs. Alguns roteadores dentro do AS são escolhidos como roteadores responsáveis (RR), onde cada RR recebe um Publisher Identifier (PID) e mantém uma tabela que contém seus publicadores. O RR armazena em cache apenas os conteúdos associados aos publicadores que estão ligados a ele. O CoRC propõe que um pequeno número de conteúdo seja guardado em algum nó NDN da rede, pois a criação de FIBs escaláveis ainda é um problema. Cada publicador coloca no conteúdo publicado o PID de seu RR, como sendo a primeira parte do nome publicado e a localização do publicador acontece via RR.

\section{Programming Protocol-Independent Packet Processors}

A linguagem P4 permite modelar switches baseados em Protocol Independent Switch Architecture (PISA) e projetados para categorizar as funções do plano de dados. Esta 
foi concebida nas ideias de SDN, pois algumas de suas implementações foram usadas no protocolo OpenFlow. Ademais, o $\mathrm{P} 4$ tem por objetivo realizar o encaminhamento de pacotes através de um código aberto e flexível para realizar o encaminhamento das mais diferentes implementações, protocolos e abordagens que venham a serem desenvolvidas para a Internet.

A quantidade de nós finais está crescendo exponencialmente. Normalmente estes são conectados a um dispositivo de borda que está conectado a um núcleo de rede no qual normalmente utiliza MPLS, que distribui etiquetas para simplificar a troca de informações dentro do núcleo. No entanto, o crescimento do número dos dispositivos finais torna esta tarefa de distribuição de inúmeros labels árdua. Além da distribuições de labels, a quantidade de headers diferentes em uso estão se proliferando exponencialmente [Bosshart 2014]. Como o plano de controle não está totalmente desacoplado, não existe uma maneira fácil de modificar os formatos dos pacotes e adicionar ou remover novas características. Assim, é necessário realizar alterações nos elementos de encaminhamento e nos controladores. Com o aumento do uso de linguagens de programação para realizar alterações no plano de dados, como o P4, torna-se possível a alteração da forma que os comutadores processam os pacotes após a implementação.

\section{Proposta de ICN com NovaGenesis em P4}

Por se tratar de uma arquitetura que adota premissas como armazenamento de dados em cache, a flexibilidade da nomeação de dados e NBs significativos entre as suas entidades, a NG tem potencial para incorporar ingredientes chaves das ICNs. Atualmente, o roteamento NG está baseado em tuplas que especificam o host, o processo, o sistema operacional e o serviço a que um pacote está destinado. No entanto, pode-se criar uma nova modalidade que se baseie apenas em labels, similar a label switching, e, assim, estabelecer uma mecânica similar ao NDN com tabelas PIT, FIB e nonces para disponibilizar o conteúdo em cache. Com isto, permite-se a replicação de dados ao longo da rede e possibilita que um consumidor obtenha o conteúdo mais próximo de sua localidade.

Ademais, a NG é uma arquitetura que atualmente está participando do projeto Future Internet Exchange Point [J. Gavazza 2019] fomentado pela FAPESP, sob a chamada \#2015/24518-4, e também pela CAPES. Sob esta proposta de trabalho, visa-se o desenvolvimento de um hardware capaz de processar e encaminhar pacotes de maneira flexível e independente do protocolo que está sendo empregado. Por meio deste trabalho, demonstra a viabilidade de empregar um hardware programável, flexível e dinâmico para o roteamento de pacotes NG, ETArch e TCP/IP.

A Figura 1 ilustra uma proposta de trabalho que possibilite tal convergência. Nesta, o nó "Host 1" armazena os diversos conteúdos Name Resolution and Networking Cache Service representando um cache de rede NG. Este é composto pelo PSS, responsável por implementar o modelo de comunicação pub/sub e disponibilizar o conteúdo à rede, o Generic Indirect Resolution Service (GIRS), o qual recebe de fato as demandas do PSS e faz o encontro entre estas solicitações e os dados armazenados em hash tables, e o HTS, responsável por armazenar os conteúdos e dados diversos, similar ao CS do NDN. Por sua vez, o "Hardware P4" é modelado através da linguagem P4 e serve como um ponto de interconexão do domínio, sendo responsável por encaminhar os dados no domínio, promovendo um mecanismo similar ao CoRC ao armazenar uma tabela dos 
publicadores que estão ligados a ele. Por meio deste, pode-se lidar com uma solicitação distinta e promover a entrega do conteúdo desejado, similar ao NDN que se baseia em PIT e FIB. Por fim, tem-se o "Host 2" que é outro dispositivo distinto que faz a solicitação de um conteúdo à rede, sendo o interesse publicado pela combinação do NRNCS e o PGCS.

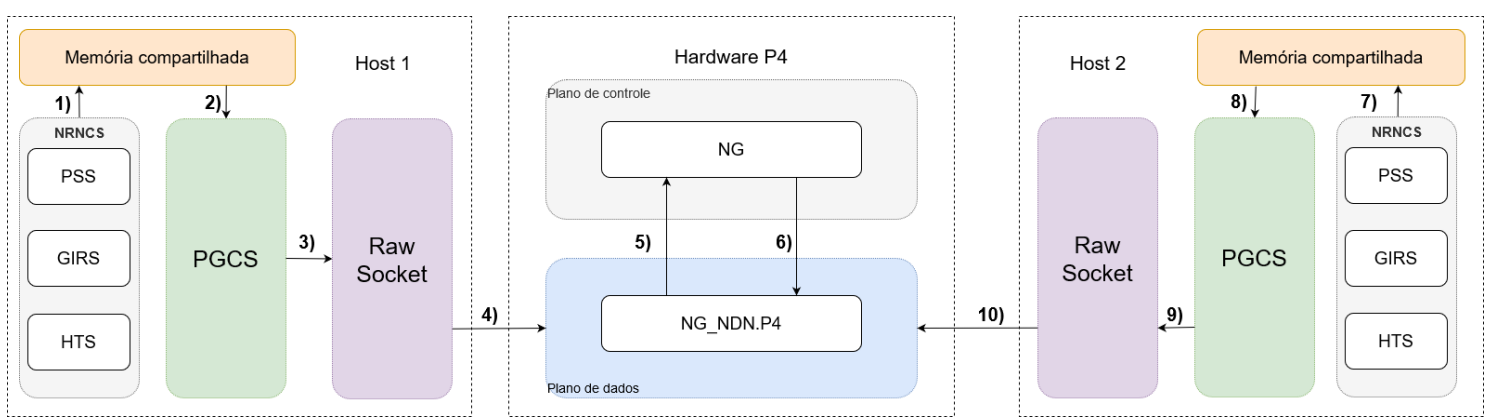

Figura 1. llustração da proposta de trabalho com os conceitos de NG, NDN e P4.

Um nó que tenha a característica de ser um cache de rede expõe o seu conteúdo periodicamente através de um Hello, a qual é uma mensagem padrão NG que serve para divulgar a característica de um nó. Essa mensagem é construída no Name Resolution and Network Caching Service (NRNCS), o qual possui os dados e conteúdos específicos em sua HTS e publicado por meio do PSS, sendo orientado pelo GIRS.

Cada serviço se vale da memória compartilhada do sistema para se comunicar, a qual é interface para os mais diversos serviços NG presentes. Assim, o NRNCS encaminha essa mensagem de divulgação à memória compartilhada, ilustrado pela seta 1), e o PGCS a obtém em 2). Após isto, a mensagem recebida é encapsulada a um protocolo adequado de comunicação, inserindo os cabeçalhos de roteamento, agora baseado nas premissas de NDN, e dados de controle. Em seguinda, o PGCS encaminha, 3), essa mensagem ao destino via Raw Socket, 4), o qual serve como a entrada/saída de dados do sistema.

O Hardware P4 promove o roteamento de conteúdo a nível de um domínio de rede, a mensagem enviada pelo Host 1 é recebida por este hardware, 4). Neste momento, o seu firmware "NG_NDN.P4" reconhece o protocolo NG e, 5), encaminha ao plano de controle por se tratar de uma mensagem de divulgação de conteúdo. Deste modo, o agente de controle NG reconhece que a mensagem recebida é um Hello e atualiza a FIB do dispositivo com a nova rota de um publicador conectado a este nó, 6). Como consequência, qualquer subscriber que busque um conteúdo do Host 1 é direcionado à interface de comunicação que alcance tal nó. Apenas para ilustrar a busca por um conteúdo, pode-se supor que o Host 2 possui uma demanda por algo armazenado na cache de rede. Por se tratar da arquitetura NG que mantém uma estrutura similar a qualquer dispositivo, a mensagem de interesse é gerada como o Hello, executando os passos 7), 8), 9) e 10) apresentados na Figura 1. Ao receber esta mensagem, o hardware $\mathrm{P} 4$ associa o Content ID requisitado ao destino já armazenado em sua FIB e promove a entrega do conteúdo armazenado na cache de rede ao Host 2.

\section{Conclusão}

A evolução das redes demonstra cada vez mais que a necessidade de integração de diferentes tecnologias é imprescindível para conectar tudo e todos de forma benéfica. Com 
os novos hardwares que estão sendo construídos com tecnologias que permitem a sua configuração via software, aceitando diferentes linguagens de programação e permitindo alterações em tempo de execução. Por meio destes, será mais fácil realizar a construção de redes auto-organizáveis, as quais se constroem e se modificam de acordo com a necessidade do usuário ou de uma aplicação específica. Ademais, fomentam redes nas quais protocolos e serviços serão compostos dinamicamente e terão ciclos de vida definidos, possibilitando a conexão de qualquer objeto, em qualquer lugar e a qualquer hora. Esse fenômeno tecnológico pode tornar pessoas e coisas interconectadas, provendo uma gama de novas ideias promissoras.

\section{Agradecimentos}

Este trabalho foi parcialmente financiado pela RNP, com recursos do MCTIC, processo No 01250.075413/2018-04, sob o projeto Centro de Referência em Radiocomunicações (CRR) do Instituto Nacional de Telecomunicações - Inatel, Brasil. Os autores agradecem também a FAPEMIG, CNPq, CAPES, FAPESP, SENAC CEP GUAXUPÉ e SINDISAT.

\section{Referências}

A. Alberti, M. Casaroli, A. F. S. R. (2017). Naming and name resolution in the future internet: Introducing the novagenesis approach. Future Generation Computer Systems, 67:163-179.

Bosshart, P. Daly, A. V. (2014). P4: Programming protocol-independent packet processors. ACM SIGCOMM Computer Communication Review, 44(3):87-95.

Choi, H. Yoo, T. C. (2014). Corc: coordinated routing and caching for named data networking. In Proceedings of the tenth ACM/IEEE symposium on Architectures for networking and communications systems, pages 161-172. ACM.

ICN (2017). Irtf information-centric networking research group. https://irtf.org/icnrg.

J. Gavazza, T. dos Santos, L. V. A. S. M. (2019). Implementação de um switch em p4 com suporte simultâneo a múltiplas arquiteturas de internet do futuro. In 10th Workshop de Pesquisa Experimental da Internet do Futuro (WPEIF), pages 14-19. SBRC.

K. Lei, S. Zhong, H. Z. (2018). An ndn iot content distribution model with network coding enhanced forwarding strategy for 5g. IEEE Transactions on Industrial Informatics, $14: 2725-2735$.

Li, Y. and Chen, M. (2015). Software-defined network function virtualization: A survey. IEEE Access, 3:2542-2553.

Luo, C. Wu, J. T. (2015). Name label switching paradigm for named data networking. In IEEE Communications Letters, pages 335-338. IEEE.

Mays, N. Thomos, M. R. (2016). Information-centric multilayer networking: Improving performance through an icn/wdm architecture. In IEEE Transactions on Networking, pages 83-97. IEEE.

Potys, I. Marsh, F. O. (2016). Netinf tp: A receiver-driven protocol for icn data transport. In IEEE 23rd International Symposium on Quality of Service. IEEE.

Wang, C. Alyyan, A. Z. (2012). Ospfn: An ospf based routing protocol for named data networking. Technical report, Technical Report NDN-0003. 